\title{
Primary gastric tuberculosis
}
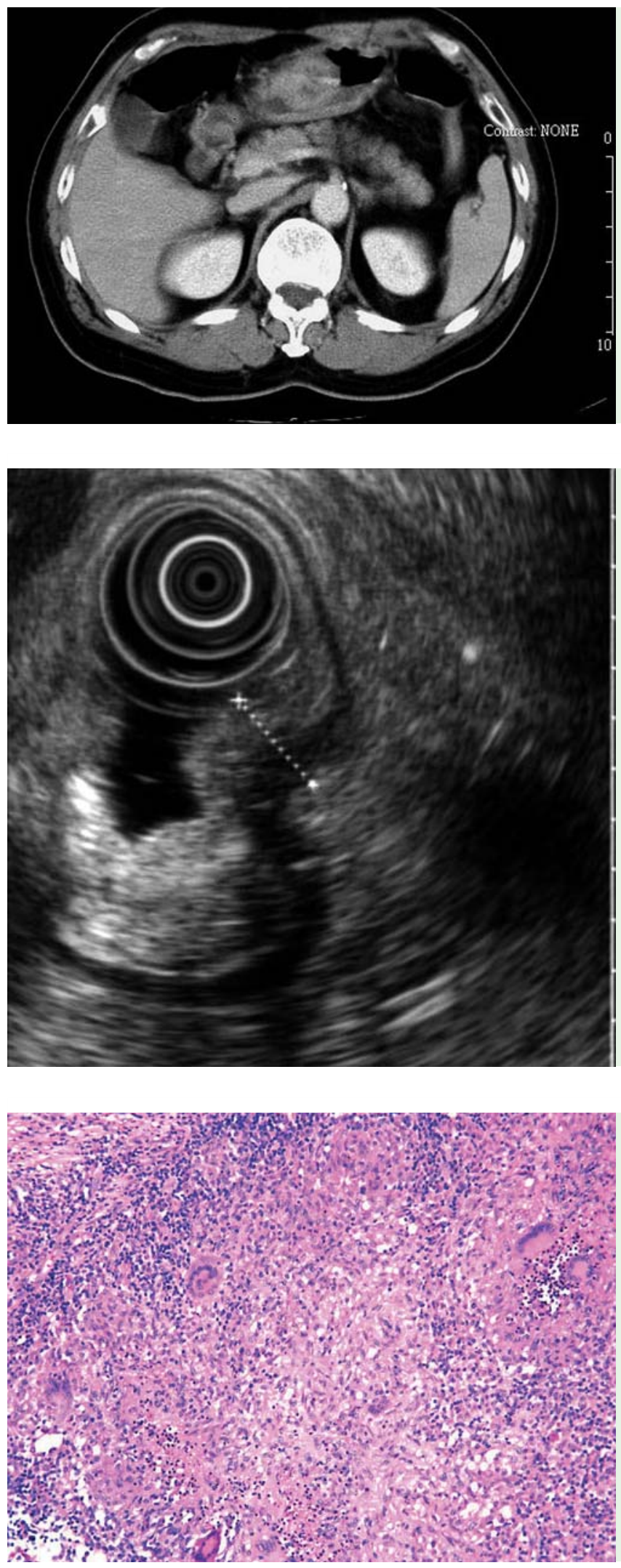

Fig. 1 Axial contrastenhanced computed tomography (CT) scan showing a homogeneous density, $4 \times 3 \mathrm{~cm}$, enhancing mass with foci of low attenuation, arising from the greater curvature of gastric antrum and infiltrating the surrounding omentum. The mass was suspected to be a gastric tumor with perifocal invasion.

Fig. 3 Upper gastrointestinal endosonography showing conspicuous thickening of the gastric wall over the lower body, up to the antrum, with infiltration of the lesion into the deep mucosa and the submucosal layer. The muscularis propria was mildly thickened.

Fig. 4 Necrotizing granulomatous inflammation involving all the layers of the gastric wall and the gastric mucosa, with ulceration. The lesion was adherent to the mesocolon. Langerhans giant cells with multiple peripherally located nuclei are seen in the granuloma.

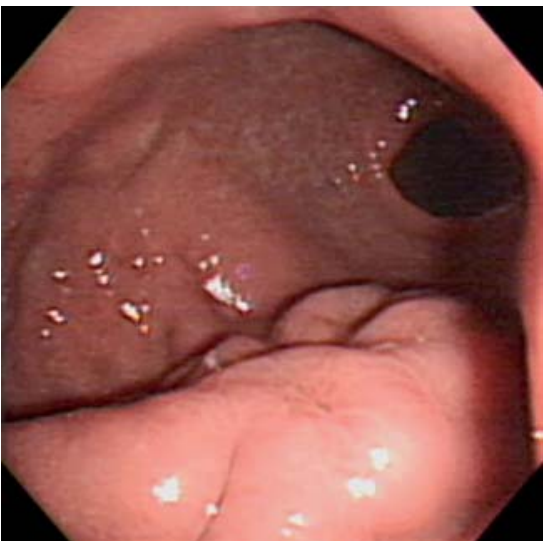

Fig. 2 Enlarged gastric fold, $3-4 \mathrm{~cm}$ in length, in the lower part of the body of the stomach and extending into the antrum on the side of the greater curvature. A small ulcer is seen on the top.

Primary gastric tuberculosis is rare, and the diagnosis depends on a high index of suspicion [1]. We present a case of gastric tuberculosis and gastric submucosal tumor with an unusual appearance in an immunocompetent adult with no pulmonary involvement.

A 68-year-old man presented with epigastric pain, which he had been having for a few months. He had been referred to us for a suspected gastric tumor following an endoscopy at another hospital. An abdominal computed tomography (CT) scan revealed a $4 \times 3 \mathrm{~cm}$ mass with homogeneous density arising from the gastric antrum and infiltrating the surrounding omentum ( Fig. 1).

Gastroscopy showed an enlarged gastric fold, about $3-4 \mathrm{~cm}$ in size, over the lower part of the body of the stomach and extending to the antrum on the side of the greater curvature, with a small ulcer on the top ( $\bullet$ Fig. 2).

Endosonography showed the lesion extending into the deep mucosa and the submucosal layer of the thickened gastric wall. The muscularis propria was mildly thickened ( $\bullet$ Fig. 3).

The patient underwent subtotal gastrectomy and segmental resection of the transverse colon because the lesion was adherent to the mesocolon and the transverse colon, mimicking a localized malignancy. Histopathological examination of the surgical specimen revealed necrotizing granulomatous inflammation with foci of Lan- 
gerhans giant cells, involving the whole gastric wall. ( $\mathbf{F i g}$.4) Acid-fast staining did not reveal any acid-fast bacilli, but a polymerase chain reaction (PCR) test for tuberculosis was positive. Accordingly, a diagnosis of gastric tuberculosis was made.

The patient's condition stabilized post operatively and he was discharged.

Tuberculosis may involve any part of the gastrointestinal tract, in particular, the ileocecal region [2], and mostly occurs secondary to pulmonary or other systemic tuberculosis. Primary, isolated gastric tuberculosis is rare, and gastric tuberculosis sometimes mimics gastric cancer, [3] which occurs in about $10 \%$ of gastric tuberculosis cases [4]. If gastric tuberculosis is clinically suspected, PCR test of the biopsy specimen provides a faster, alternative route for diagnosis while excluding Crohn disease with $100 \%$ specificity and $27 \%-75 \%$ sensitivity [5].
Endoscopy_UCTN_Code_CCL_1AB_2AD_3AF

P. F. Liu, C. S. Chang, J. Wang, C. C. Wu, H. Z. Yeh

Division of Gastroenterology, General

Surgery and Pathology, Taichung Veterans

General Hospital, Taichung, Taiwan

\section{References}

1 Lin OS, Wu SS, Yeh KT et al. Isolated gastric tuberculosis of the cardia. J Gastroenterol Hepatol 1999; 14: 258-261

2 Marshall JB. Tuberculosis of the gastrointestinal tract and peritoneum. Am J Gastroenterol 1993; 88: 989-999

$3 \mathrm{Kim}$ SE, Shim KN, Yoon SJ et al. A case of gastric tuberculosis mimicking advanced gastric cancer. Korean J Intern Med 2006; 21 : 62-67

4 Chowdhary GN, Dawar R, Misra MC. Coexisting carcinoma and tuberculosis of stomach. Indian J Gastroenterol 1999; 18: 179-180

5 Lau CF, Wong AM, Yee KS et al. A case of colonic tuberculosis mimicking Crohn's disease. Hong Kong Med J 1998; 4: 63-66
Bibliography

DOI $10.1055 / \mathrm{s}-0029-1215001$

Endoscopy 2009; 41: E327 - E328

(c) Georg Thieme Verlag KG Stuttgart · New York . ISSN 0013-726X

\section{Corresponding author}

\section{H. Z. Yeh, MD}

Division of Gastroenterology

Taichung Veterans General Hospital

No. 160 , Sec. 3

Chung-Kang Road

Taichung

Taiwan

R.O.C. 40705

Fax: +886-4-23595046

hzen.yeh@msa.hinet.net 